\title{
Improved lovastatin production by inhibiting (+)-geodin biosynthesis in aspergillus terreus
}

\begin{abstract}
Lovastatin is widely prescribed to reduce elevated levels of cholesterol and prevent heartrelated diseases. Cultivation of Aspergillus terreus (ATCC 20542) with carbohydrates or lowvalue feedstocks such as glycerol produces lovastatin as a secondary metabolite and (+)geodin as a by-product. An A. terreus mutant strain was developed (gedC $\Delta$ ) with a disrupted (+)-geodin biosynthesis pathway. The gedC $\Delta$ mutant was created by inserting the antibiotic marker hygromycin B (hyg) within the gedC gene that encodes emodin anthrone polyketide synthase (PKS), a primary gene responsible for initiating (+)-geodin biosynthesis. The effects of emodin anthrone PKS gene disruption on (+)-geodin and lovastatin biosynthesis and the production of the precursors acetyl-CoA and malonyl-CoA were investigated with cultures based on glycerol alone and in combination with lactose. The gedC $\Delta$ strain showed improved lovastatin production, particularly when cultivated on the glycerol-lactose mixture, increasing lovastatin production by $80 \%(113 \mathrm{mg} / \mathrm{L})$ while simultaneously inhibiting (+)-geodin biosynthesis compared to the wild-type strain. This study thus shows that suppression of the (+)-geodin pathway increases lovastatin yield and demonstrates a practical approach of manipulating carbon flux by modulating enzyme activity.
\end{abstract}

Keyword: (+)-Geodin; Aspergillus terreus; Emodin anthrone polyketide synthase; Gene knockout; Lovastatin 